\title{
LA USABILIDAD PERCIBIDA DE LOS CHATBOTS SOBRE LA ATENCIÓN AL CLIENTE EN LAS ORGANIZACIONES: UNA REVISIÓN DE LA LITERATURA
}

\author{
Oswaldo Daniel Casazola Cruz \\ odcasazolac@unac.edu.pe \\ https://orcid.org/0000-0003-2521-530X \\ Gianfranco Alfaro Mariño \\ galfarom@unac.edu.pe \\ JAIME BuRgos TEJADA \\ jburgost@unac.edu.pe \\ Omar Augusto Ramos More \\ oaramosm@unac.edu.pe \\ Universidad Nacional del Callao, Perú
}

\section{Resumen}

Un chatbot es una aplicación de software basada en inteligencia artificial que permite simular una conversación con una persona. Sin embargo, a pesar de sus múltiples beneficios, una cantidad sustancial de chatbots luchan por satisfacer a los usuarios. El objetivo de este artículo fue el de describir la usabilidad percibida de los chatbots sobre la atención al cliente en distintas organizaciones. Por ello, se ha realizado una revisión sistemática de la literatura, con base en datos de. Google Académico y EBSCO, en el período comprendido entre el 2015 y el 2020, para analizar las evidencias recogidas en diferentes investigaciones sobre: (a) la naturaleza de los chatbots, (b) su implementación, (c) la usabilidad percibida y (d) el auge de esta tecnología.

PALABRAS CLAVE: chatbot / agente conversacional / asistente virtual / atención al cliente / usabilidad 
PERCEIVED USABILITY OF CHATBOTS ON CUSTOMER SERVICE IN ORGANIZATIONS: A REVIEW OF THE LITERATURE

\section{Abstract}

A chatbot is a software application based on artificial intelligence, which allows to simulate a conversation with a person. However, despite the many benefits, a considerable number of chatbots struggle to satisfy users. The objective of this article was to describe the perceived usability of chatbots in customer service in different organizations. Therefore, the current study performed a systematic review of the literature on Google Scholar and EBSCO data, between the period 2015 and 2020, to analyze the existing evidence regarding (a) the nature of chatbots, (b) its implementation, (c) perceived usability, and (d) the rise of this technology.

KEYWORDS: chatbot / conversational agent / virtual assistant / customer servic / usability 


\section{INTRODUCCIÓN}

En el ámbito mundial, las organizaciones buscan mejorar la atención al usuario, dado que los requerimientos de sus usuarios han ido en aumento con el tiempo. La interconectividad de las emergentes redes sociales otorga nuevas formas de comunicarnos y nuevas oportunidades de brindar atención a los clientes (Condori, 2017). Sin embargo, ¿qué sucede cuando nuestros interlocutores son chatbots, bots desarrollados para imitar la interacción humana? ¿Cómo percibimos un estilo de comunicación que es tan similar al nuestro, pero diferente? Gutiérrez (2019) sostiene que, a diferencia de los humanos, la comunicación de los chatbots se basa en la computación cognitiva, la cual implica sistemas de autoaprendizaje con el fin de simular el comportamiento humano. Por otra parte, estos sistemas se encuentran en constante evolución y mejora debido a la naturaleza volátil del lenguaje natural, lo cual hace que la recuperación o procesamiento sea difícil (Ascencio, 2019). Sin embargo, la posibilidad de que los usuarios interactúen con la tecnología es lo que hace que los chatbots sean tan atractivos. El uso del lenguaje natural como modo de interacción en un chatbot puede hacer que la tecnología sea mucho más accesible y menos complicada para los usuarios (Expósito, 2019; García-Reina 2018). Por ello, el presente artículo considera importante estudiar las formas en que los humanos perciben la interacción con los chatbots.

Algunos hallazgos en la revisión de la literatura evidencian que el concepto de chatbot posee múltiples términos usados para referirse a él. Estos son empleados a disposición del autor y principalmente son: Agente conversacional, Entidad conversacional, Bot conversacional, Asistente virtual, Agente virtual, Asesor virtual (Condori, 2017; Durán, 2015; Jimenez, 2019; Pérez, 2019).

Los agentes conversacionales son sistemas diseñados para comunicarse con los usuarios mediante el uso del lenguaje natural. Si bien la interacción suele establecerse principalmente a través de mensajes de voz (audio) o mediante la escritura (texto), también se puede hacer uso de otras modalidades de la comunicación integrando gráficos, imágenes o animaciones, que pueden contribuir a la construcción de entornos altamente interactivos (Gomez, 2019). Originalmente, la propuesta de un chatbot es crear la sensación de una conversación real a través de un software debidamente programado para tal tarea. Están diseñados para mantener una conversación trivial y utilizan un enfoque de estímulo-respuesta en el que la entrada de los usuarios se compara con un gran conjunto de patrones almacenados para generar una respuesta (Limón, 2016). Adicionalmente a esto, Zarabia (2018) señala la facilidad con la que los chatbots pueden incorporarse en cualquier sector debido a su disponibilidad y fácil usabilidad, y da como ejemplo los campos de aplicación empresarial, personal, académico y de entretenimiento como los principalmente usados por los chatbots. 
En el ámbito organizacional, este concepto se encuentra relacionado con el proceso de información y conocimiento para la toma de decisiones; la inteligencia artificial (IA) permite optimizar tiempos, mano de obra y mejorar el servicio (Estrada, 2018). Según Martínez (2019) la inteligencia artificial está captando la atención de las empresas por la forma de procesar el lenguaje natural y el aprendizaje automático (machine learning), la implementación de un asistente virtual chatbot cuenta con el soporte adecuado para atender las conversaciones con los clientes ya que podrá trabajar de manera independiente sin la necesidad de contar con un operador físico en el canal de comunicación, brindándoles una mejor experiencia e inmediatez a los datos, disponibilidad de tiempo las 24 horas, teniendo así un mayor rendimiento para conseguir los objetivos planteados de marketing y negocios con los clientes, donde será de gran ayuda la asistencia automatizada del mismo. Por otro lado, Abata y Ramírez (2018) en su investigación indican que del nivel de madurez empresarial dependerá el desempeño del agente; el asistente virtual no podrá ser puesto en producción si la empresa no cuenta al menos con una base de datos o archivos digitales de los cuales se pueda obtener información.

La revisión de literatura ha mostrado la relación favorable existente entre la atención al cliente y el uso de chatbots. Según Burgos y Saavedra (2019) en un estudio que tiene como objetivo determinar en qué medida el uso de un chatbot mejora el proceso de ventas, señalan una influencia positiva de parte del uso de un chatbot y una percepción de agrado por los usuarios; es decir, al obtener respuestas adecuadas y de manera instantánea, los usuarios del servicio se encuentran satisfechos. Asimismo, distintos autores también aseguran que el uso de la entidad conversacional para la atención a los usuarios tiene una correlación significativamente alta en la percepción de la calidad de atención brindada por la organización, al proporcionar la información de manera rápida y eficiente, superando la lentitud de búsqueda manual de la información por parte de los usuarios (Jimenez, 2019; Busqué, 2018; Peralta, 2018). Sumado a esto, Choque (2018) señala que en un canal de consulta, la forma y tono de respuesta son vitales, pues si no se toma en cuenta generaría frialdad y lejanía para con sus usuarios. Lo que resultaría en que los usuarios no usaran este canal.

Según lo anterior, es favorable implementar un chatbot en una organización a futuro. Respecto a esto, Santos (2018) indica que se espera un auge de su uso en los próximos años. Los usuarios los prefieren en las búsquedas y en el acceso a los contenidos y servicios digitales. Además, los bots pueden dispensar un trato personalizado que se mejora según el bot aprende con la experiencia adquirida y con el conocimiento de las preferencias del usuario. Agregando a esto, Garibay (2020) señala que, con el actual auge de las apps, los chatbots y dichas herramientas pueden ser fusionadas para brindar un mejor servicio al cliente. Plataformas como WhatsApp o Facebook se están convirtiendo rápidamente en las interfaces predilectas de comunicación para la atención al consumidor, debido a que millones de usuarios las utilizan. 
De esta manera, se evidencia que la demanda de chatbots también está creciendo y debido a esta demanda, las áreas de aplicación también se están expandiendo (educación, servicio al cliente, comercio electrónico, salud, música, etcétera). Esto crea nuevos requisitos que empujan a los chatbots a ampliar aún más sus límites e incorporar características de otros tipos de interfaces de conversación. Actualmente, muchos chatbots despliegan técnicas más complejas, como incorporar un avatar, sintetizador de voz, animación hablada, entre otros (Hurtado y Zúñiga, 2019). Así, el objetivo de esta investigación fue describir la usabilidad percibida de los chatbots sobre la atención al cliente en las organizaciones a partir de la revisión de la literatura científica de los últimos cinco años, para definir la naturaleza de los chatbots y su influencia sobre la atención al cliente en las organizaciones.

\section{MATERIALES Y MÉTODOS}

\subsection{Tipo de estudio}

Se realizó una revisión sistemática de la literatura científica con base en la adaptación de la metodología Prisma (Preferred Reporting Items for Systematic Reviews and MetaAnalyses) (Urrútia y Bonfill, 2010). La pregunta de investigación establecida para conducir el proceso metodológico fue la siguiente: ¿Cuál es la usabilidad percibida de los chatbots sobre la atención al cliente en las organizaciones descritos en la literatura científica?

\subsection{Fundamentación de la metodología}

La revisión de la literatura científica es una estrategia de recopilación de información que emerge ante la necesidad de conocer de manera sintética los resultados de las investigaciones. Las revisiones sistemáticas intentan reunir todo el conocimiento de un área específica, resaltando lo que se sabe sobre un tema específico, a través de los resultados obtenidos en diferentes estudios y así ofrecer recomendaciones para futuras prácticas e investigaciones (Urrutia y Bonfill, 2010). En la revisión sistemática que se presenta en este artículo, se analizó y sintetizó la evidencia encontrada en las investigaciones.

\subsection{Proceso de recolección de información}

Para garantizar la sensibilidad del proceso de búsqueda se definieron como descriptores los siguientes términos a partir de la pregunta de investigación: "Chatbot", "Agente conversacional", "Asistente virtual", “Entidad conversacional”, “Atención al cliente". Por especificidad de la búsqueda de la literatura científica, se diseñó un protocolo con la combinación de los términos establecidos y los operadores booleanos: [("Chatbot" OR "Agente conversacional" OR "Asistente virtual" OR "Entidad conversacional") AND ("atención al cliente")]. Igualmente se definió como base de datos especializada para la búsqueda a EBSCO; y como base de datos multidisplinaria se eligió Google Académico. 
- EBSCO:

- [("Chatbot" OR "Agente conversacional" OR "Asistente virtual" OR "Entidad conversacional") AND ("atención al cliente")]

- Google Académico

- [(“Chatbot" OR "Agente conversacional" OR "Asistente virtual” OR "Entidad conversacional")]

Los artículos seleccionados se importaron al software de gestión de referencias Mendeley, para su gestión en la eliminación de citas duplicadas.

\subsection{Criterios de inclusión y de exclusión}

Se incluyeron artículos originales publicados en bases de datos científicas como EBSCO y Google Académico, en idioma español o inglés, entre los años 2015-2020, que describieran a los chatbots sobre la atención al cliente en las organizaciones.

La revisión se centró en los estudios que incluyen hallazgos y teorías sobre factores que pueden influir potencialmente en la usabilidad percibida de los chatbots sobre la atención al cliente en organizaciones y estudios que incluyen métodos de evaluación que podrían informar sobre los criterios utilizados durante la evaluación.

Como criterio de exclusión se tomó en cuenta cualquier artículo que no se encontrara en idioma español o inglés.

El protocolo de búsqueda y de extracción de información fue aplicado por tres revisores de forma independiente, cuyas diferencias fueron analizadas y resueltas por mutuo acuerdo.

\subsection{Proceso de revisión}

Todos los artículos referenciados a través de los descriptores fueron analizados y codificados independientemente por tres investigadores, quienes realizaron por lo menos dos lecturas completas de los mismos.

Para analizar la producción científica en relación con la usabilidad percibida de los chatbots sobre la atención al cliente, se agruparon los trabajos en cuatro áreas de relevancia en la literatura sobre chatbots: (1) sobre su naturaleza, (2) sobre su implementación, (3) sobre la usabilidad percibida y (4) sobre el auge de esta tecnología. Los criterios de clasificación surgen del estudio de la revisión sistemática. 


\section{RESULTADOS}

Se encontraron treinta y ocho artículos que respetaban los criterios de inclusión indicados. Los resultados derivados de la revisión se describen a continuación según las preguntas previamente mencionadas. Como algunos trabajos respondían a más de una pregunta, la decisión de incluirlo en un grupo fue consensuada entre los investigadores. Por razones de parsimonia y de claridad, se discutirán los diferentes trabajos organizados en cada pregunta.

\subsection{Sobre la naturaleza de los chatbots}

Se encontraron cinco artículos centrados en la naturaleza de los chatbots, aportando explicaciones conceptuales y metodológicas sobre los diferentes componentes que los integran. El artículo de Herrero y Varona (2018) aborda la temática del fenómeno de los bots y los chatbots como herramientas de interacción en los medios de comunicación. También resaltan el funcionamiento de los chatbots sin recurrir a instalar algún programa o aplicativo; en cambio, utilizan los recursos de otras más universales como Whatsapp, Telegram o Facebook Messenger. Por otro lado, señalan el reto que tienen los chatbots para seguir mejorando su forma de interactuar, para así lograr un servicio más natural y cercano con sus usuarios.

En la investigación de Gutiérrez (2019) se buscó explorar el mundo de los chatbots, herramientas y técnicas de desarrollo. Resalta las múltiples aplicaciones y entornos en donde se utilizan los chatbots como: educación, entretenimiento, ventas, turismo, entre otros. Analiza la estructura de los chatbots y expone que estos sistemas se miden en base a la usabilidad y satisfacción del usuario con su uso. Por último, el autor se centra en el funcionamiento de los chatbots y plataformas de desarrollo de estos como IBM Watson, Dialogflow, Rasa Stack y Flow Xo.

Gomez (2019) analizó la viabilidad del uso de técnicas de procesamiento del lenguaje natural y de aprendizaje automático para naturalizar y asistir el proceso de búsqueda de trabajo a través de medios digitales. El autor sostiene que un agente conversacional conseguirá guiar de forma efectiva el proceso de extracción de información a la vez que consigue mejorar la usabilidad del sistema y la experiencia del usuario al naturalizar la comunicación. El uso de técnicas de procesamiento del lenguaje natural resulta fundamental para la extracción de información relevante sobre los datos de entrada. Por este motivo, el autor defiende que las técnicas de aprendizaje son de gran utilidad al captar patrones y estructuras en los datos, puesto que habría resultado inviable capturar la complejidad y la casuística que puede llegar a darse en los datos de entrada mediante programación basada en reglas.

El trabajo de Limón (2016) busca responder a la pregunta, ¿es posible realizar consultas de datos en forma fácil, rápida, útil y específica para una organización? Se 
resalta la gran importancia de los datos y de cómo se puede utilizar un chatbot para la interpretación y consulta de información. Analiza los chatbots como interfaz en la comunicación humano-computador. Destaca como las principales virtudes de chatbot: exactitud, eficacia, eficiencia, innovador, rápido, sencillo y útil para tomar decisiones. Señala que un chatbot es una herramienta hija de las ciencias exactas y la lingüística, con la capacidad de reconocer sistemáticamente por su cualidad natal las intenciones de quien lo utiliza; es el perfecto intérprete para realizar el vínculo que facilitaría la consulta ya no solo de datos sino de información, capaz de ayudar a encontrar relevancia en datos almacenados.

El trabajo de Abata y Ramírez (2018) se enfocó en el desarrollo de un prototipo de asistente virtual que entregue conocimiento de una empresa de forma más organizada y precisa. Los autores expresan su deseo de crear un asistente virtual con la capacidad de aprender sobre lo que el usuario está preguntado. Para ello realizan un análisis de diversas tecnologías para el desarrollo de un asistente virtual, haciendo uso de distintas tecnologías como Bot Framework, el cual posee un SDK para crear un ambiente de interacción humano-computador apoyándose en NodeJS, .NET para la creación de servicios que interactuaran con Bot Framework en Azure de Microsoft debido a que permite un ambiente de alta disponibilidad y escalabilidad, haciendo uso del formato JSON para la comunicación de datos mediante el protocolo HTTPS, concluyendo que esto es lo más oportuno para desarrollo y posterior implementación en una organización.

\subsection{Como implementar un chatbot}

Se encontraron dieciséis artículos centrados en la implementación de un chatbot, explicando las actividades realizadas para la implementación de la entidad conversacional en las organizaciones. Estos artículos se pueden clasificar en tres grupos, según el objetivo central de los mismos. Un primer grupo de siete artículos describe la posibilidad de implementar un chatbot en un entorno académico. Un segundo grupo, también de siete estudios, se centró en la implementación de un agente conversacional en una empresa. Y, por último, un tercer grupo compuesto de dos trabajos se enfocó en el estudio de la implementación de un chatbot en los sectores de salud y turismo.

Referente al primer grupo sobre el entorno académico, Cubero (2015) y Vallejo (2015) en sus investigaciones abordan la implementación de un chatbot para la web de la Facultad de Informática de la Universidad Complutense de Madrid. Se expone la dificultad de implementar un chatbot en el entorno web de la universidad dada la dependencia de este, la importancia de que el agente esté correctamente integrado y sea de fácil acceso y uso. Además, detallan la importancia de escoger la tecnología correcta de desarrollo de la entidad conversacional según el objetivo del proyecto propuesto. También resaltan el valor del aprendizaje del chatbot para comunicarse con sus usuarios. En el trabajo 
de Abbati y Moreno (2019) se plantea la implementación de un asistente virtual para la biblioteca de Universidad Complutense de Madrid. El autor señala las ventajas de desarrollar el asistente virtual en un entorno de código abierto frente a las plataformas de desarrollo prediseñadas como IBM Watson que limitan funciones en una suscripción gratuita. También expone la importancia de la unificación de la información para el funcionamiento correcto del asistente virtual y la satisfacción de los usuarios. Por último, resalta la importancia de que este asistente virtual se encuentre desarrollado en forma de aplicativo móvil tanto para iOS y Android, con la finalidad de que sus usuarios tengan una mayor accesibilidad de la información. La investigación de Expósito (2019) también abordó el tema de la implementación de un asistente virtual para la biblioteca de una universidad. El autor señala la importancia de que el asistente virtual sea capaz de desenvolverse de forma cómoda y natural, a través de opciones como el funcionamiento en el idioma inglés y el reconocimiento de voz. También expone la relevancia que tiene darle un adecuado entrenamiento al asistente para que sea capaz de manejar múltiples situaciones, puesto que pueden presentarse errores en frases que poseen estructuras muy parecidas. Por último, manifiesta su preferencia por la herramienta de desarrollo de IBM Watson y sus tecnologías para la implementación. En el trabajo de Manjarrés y Echeverri (2020) se plantea la implementación de un asistente virtual académico utilizando tecnologías cognitivas del procesamiento de lenguaje natural. El autor realiza un análisis de las plataformas de chatbot más relevantes y se decanta por Dialogflow, señala que se debe tener claro el público objetivo, el tipo de lenguaje que usa y el carácter del agente, ya que la experiencia de usuario en un chatbot viene definida por la fluidez de la conversación. Además, resalta también la importancia del entrenamiento del bot y la integración con diferentes plataformas de mensajería como Whatsapp. La investigación de García-Reina (2018) se centra en el desarrollo de un chatbot para el servicio de atención en un colegio. El autor señala que se debe realizar un correcto estudio de la organización con la finalidad establecer el flujo que debe seguir el chatbot para su eficiente funcionamiento. Indica respecto al chatbot, que la investigación realizada sobre procesamiento de lenguaje natural y técnicas de aprendizaje automático fue fundamental para determinar el tratamiento de los textos recibidos antes y después de realizar el entrenamiento del sistema, con esto se logró caracterizar un proceso que permitiera extraer la información más relevante del contenido del mensaje. De esta manera y con la técnica de la validación cruzada, le fue posible obtener un clasificador capaz de predecir las áreas para las cuales deben ser remitidos los mensajes. El trabajo de Guijarro (2020) aborda el desarrollo de un asistente virtual para el sistema de información de una biblioteca virtual. El autor basa la construcción de su asistente en dos módulos: Backend y Frontend, desarrollando ambas partes de forma simultánea. El estudio de Pérez (2019) plantea el desarrollo de un asistente virtual para sitios web. El autor resalta la importancia de que el asistente haga uso de métodos de transcripción de audio a texto y viceversa, enviando las respuestas por escrito y por voz. 
En el segundo grupo enfocado en la implementación de agentes conversacionales en empresas, la investigación de Aguilar y Balbín (2017) plantea la implementación de un chatbot en la Superintendencia de Banca y Seguros. El autor sugiere que el uso de este chatbot estaría mayormente dirigido a una población joven que es activa con las redes sociales, por lo cual establece que el chatbot debe funcionar a través de la aplicación Facebook Messenger por ser una de las más utilizadas. Se proyecta inicialmente enfocarlo en los servicios y contenidos más demandados por los usuarios. Además, resalta la importancia de la configuración de sinónimos y listas negras para evitar que los usuarios, mientras utilizan el chatbot añadan vocabulario y formas de respuesta que no se desean. El trabajo de Martínez (2019) busca la automatización de la atención al cliente por medio de un chatbot. El autor muestra preferencia por la herramienta de desarrollo Dialogflow con la cual espera tener una mejor comunicación con sus usuarios. Sostiene que el uso del alojamiento virtual con el diseño de la base de datos logró facilitar el manejo y procesamiento de los datos que son usados por el Asistente Virtual. Por último, recomienda tomar en cuenta la integración de más redes sociales para abarcar una mayor población de usuarios. El estudio de Pérez (2019) aborda la implementación de un agente conversacional para un negocio de repuestos automotrices. El autor toma como herramienta de desarrollo Dialogflow y busca integrarlo con Facebook. Evidenció en gran magnitud la limitación latente del uso del webhook de Facebook. Asimismo, considerando que muchos elementos que soporta Facebook Messenger nativamente, no las soporta Dialogflow, explica que lo mejor es implementar un webhook en un webserver propio para poder enviar respuestas con variables y consultas hacia APIS de terceros, como Firebase o Google Custom API. El trabajo de Ascencio (2019) plantea el diseño de un chatbot para mejorar la calidad de servicio al cliente. El autor recomienda analizar profundamente la arquitectura empresarial a fin de dar un correcto diseño del chatbot y sus funciones para con los usuarios. Proyecta que el chatbot brindará grandes beneficios a la organización y también recomienda expandirse a más canales de red social a fin de llegar a más público. La investigación de Cevallos y Dela (2017) propone desarrollar un aplicativo web para automatizar los procesos rutinarios que complementen el servicio del cliente con la implementación de la tecnología de bots. Los autores afirman que el chatbot ayudará a la empresa a satisfacer todas las necesidades del cliente, evitando la insatisfacción del cliente por una mala atención. Además, se podrá reducir el personal que atiende a los clientes y eliminar el exceso de personal, transfiriéndolos a nuevas áreas de trabajo donde se desempeñaran mejor y podrán generar mayores recursos para la organización.

El tercer y último grupo analiza la implementación de un chatbot en otros sectores. El estudio de Aquino et al. (2019) proponía una solución de chatbot aplicado a los procesos agudos en el sector salud. El autor busca crear un chatbot que pueda responder a problemas de salud de baja complejidad, dándole al usuario respuestas que le permitan 
tener conocimiento básico e importante para poder proceder de forma más segura en situaciones de salud muy frecuentes como resfríos, golpes, malestares estomacales, indigestión, etc. Explica que la innovación y desarrollo permanente para la mejora continua del servicio, desempeñará un rol clave en el modelo de negocio y la atracción de cada vez más clientes, teniendo la posibilidad de añadir aspectos adicionales como servicios de enfermeras, ambulancias, asesorías psicológicas, alquiler de equipos para pacientes de la tercera edad, etc. Los trabajos de Borges (2018) y Santos (2018) pretenden diseñar un asistente virtual para recomendar sitios turísticos de interés. Proponen que para obtener un chatbot de cultura y ocio es necesario definir bien el ámbito de las funcionalidades y las acciones que se quieren cubrir en estos dominios. Indican que para el caso de turismo se observaron limitaciones al obtener la información, por la información dinámica que se puede encontrar en diferentes localidades, por lo que optó por desarrollar el asistente virtual de preguntas y respuestas sin la necesidad de que se realicen búsquedas en un sistema externo.

\subsection{Sobre la usabilidad percibida por parte de los chatbots}

Se encontraron doce artículos centrados en los resultados de implementar un chatbot en organizaciones.

El trabajo de Condori (2017) busca mejorar el servicio de atención al cliente por medio de un chatbot. El autor concluye, mediante encuestas de valoración de sus usuarios, que el implementar un asistente virtual influye de manera positiva en el servicio de atención al cliente, brinda respuestas oportunas y exactas a los usuarios; producto de esto, reduce de manera significativa los tiempos de respuesta y logra la aceptación de los usuarios.

La investigación de Busqué (2018) plantea desarrollar un asistente para los estudiantes de la Facultad de Informática de Barcelona. El autor indica que el modelo implementado de chatbot rinde muy bien, especialmente cuando las preguntas son sencillas y directas. Para preguntas más complicadas donde hay menos información relevante (es decir, preguntas donde un pequeño porcentaje de las palabras indica la intención) tiende a fallar más, pero señala que un $60,7 \%$ de las veces que el clasificador de intenciones falla, la segunda intención con más confianza es la correcta. Concluye que el uso del chatbot es muy eficaz para proporcionar la información solicitada por sus usuarios.

El estudio de Durán (2015) propone el diseño e implementación de un asesor virtual en un portal web. El autor se enfoca en medir el "nivel de contacto con el cliente" y el "nivel de satisfacción del cliente" del asesor virtual. Señala respecto al primer indicador, que el número de visitas por día se incrementa en promedio un 48 \% y que la duración de la visita se incrementa en promedio un $85 \%$. Adicionalmente a esto, indica que el primer 
indicador está estrechamente relacionado con el segundo indicador por el nivel de efectividad en la resolución de dudas de los visitantes, lo cual depende de cuan robusta es la base de datos de preguntas y respuestas del asesor. Concluye que la implantación de un asesor virtual en un portal web mejora el contacto con el cliente traducido en un incremento de las ventas.

Hurtado y Zúñiga (2019) abordan el desarrollo de un asistente virtual para los estudiantes de la Escuela Politécnica Nacional. Los autores evaluaron la satisfacción y eficiencia del chatbot por parte de los usuarios, obteniendo resultados favorables respecto a la aceptación del chatbot y eficacia de las respuestas entregadas, donde solo el 10 \% indicó no estar conforme con las respuestas del chatbot. No obstante, los usuarios reportaron que es necesario desarrollar nuevos contextos para el chatbot debido al alcance limitado de respuestas a diversos temas de este.

El trabajo de Burgos y Huaman (2019) busca determinar en qué medida el uso de un chatbot mejora el proceso de ventas en una organización. El autor señala que al utilizar el chatbot se logra reducir el tiempo para generar una cotización: anteriormente de 2806 segundos en promedio a 614 segundos en promedio. Sumado a esto, el tiempo para dar una respuesta al cliente de 770 segundos en promedio mejoró a solo 2 segundos en promedio. Además, evaluó los niveles de satisfacción de los clientes respecto a los procesos de ventas después de implementar el chatbot, obteniendo que 43,3\% respondieron "regular" para mejorar el proceso de ventas, 56,7 \% respondió "bueno". Estos resultados le indican que están satisfechos con los procesos de ventas después de implementarse el chatbot.

La investigación de Peralta (2018) propone diseñar un chatbot para la asistencia personalizada en la obtención de título para los bachilleres de la escuela profesional de ingeniería de computación y sistemas de la UPAO. El autor expone que la cantidad de actividades realizadas en el proceso se ve reducida en un $15 \%$, demostrando la reingeniería y capacidad del chatbot para automatizar el proceso mencionado. Además, conforme a las pruebas realizadas y los datos obtenidos con el estadístico T-student, se observa una reducción de tiempo promedio de 7,05 minutos, que equivale a un 59,21 \% de su media de consulta, significando una gran diferencia entre las consultas presenciales y a través del uso del chatbot.

El estudio de Choque (2018) busca implementar un modelo de tecnología cognitiva para mejorar los servicios de soporte académico con chatbots en instituciones universitarias. El autor señala que, según los datos recopilados, comprueba que el modelo propuesto de chatbot hizo la diferencia en la interacción con el estudiante. Se logró reducir en más de un $99,9 \%$ el tiempo de respuesta esperado debido a que nuestro modelo permitía responder en menos de medio segundo a cada consulta. Además, en otra evaluación sobre la aprobación del chatbot, los resultados alcanzaron el 80 \% de aprobación. 
Estrada (2018) aborda el desarrollo de un chatbot para la gestión de requerimientos e incidentes en una empresa de seguros. El autor asegura que, de los resultados obtenidos a través de las encuestas sobre el chatbot, se puede deducir que el incremento de satisfacción con los usuarios ha dado buenos resultados. Los usuarios indican un $94 \%$ que las respuestas que les brinda el chatbot sí coinciden con sus consultas. Un $90 \%$ reporta que efectivamente recibe la información oportuna a través de está implementación.

La investigación de Zarabia (2018) propone la implementación de un chatbot para los servicios a clientes del área de finanzas de seguros equinoccial. El autor realiza una medición de la efectividad y satisfacción del chatbot con sus usuarios. Obtiene que el desempeño respecto a la efectividad es de $88,57 \%$, y la satisfacción obtiene un $80 \%$. Resultando que el desempeño global del chatbot que se obtuvo al final de la evaluación es del $83,67 \%$.

El estudio de Jiménez (2019) busca determinar el nivel de correlación entre la entidad conversacional y la calidad del servicio percibido. El autor afirma que, para el caso de la entidad conversacional de inteligencia artificial, respecto a la usabilidad, se determinó que el factor predominante es la satisfacción (37\%). De igual forma, se determinó la existencia de una correlación significativa entre las variables de estudio de 0,631, lo cual supone una correlación alta. Por ello, concluye que mejorar o ampliar características de la entidad conversacional, y hacerlo más usable, contribuirá a una mejor percepción de calidad del servicio de atención por parte de los estudiantes. Además, factores específicos del chatbot como la capacidad de respuesta, fiabilidad, seguridad y empatía del servicio obtuvieron resultados favorables de parte de los usuarios.

Garibay (2020) abordó la implementación de un asistente virtual para una aerolínea mexicana. El autor indica que la retroalimentación solicitada por el chatbot arrojó que el $48 \%$ de los participantes mostró una percepción negativa de este servicio, mientras que el $53 \%$ dijo sentirse satisfecho con respecto a esta herramienta. Sin embargo, en cuanto a la calificación que recibió el servicio dado por el chatbot, se obtuvo un promedio de 4,67 de 5 estrellas; es decir, los usuarios consideraron que recibieron una buena atención por parte del asistente virtual. Por ende, el índice de satisfacción del cliente fue del 93,33\%. Otro dato relevante fue aquel que muestra la relación entre la retroalimentación del servicio de chatbot y el HitRate, debido a que se observa que, a mayor eficiencia que brinda el bot, mayor será la probabilidad de consumo de los servicios de la empresa.

El trabajo de Díaz (2020) plantea un asistente virtual conversacional aplicado al ámbito cultural. El autor opta por evaluar la aplicación chatbot en base a su rendimiento y usabilidad, obteniendo que el 57,7 \% consideran que su uso es fácil, pero un 30,8 \% consideran que tiene un uso normal. La navegabilidad de la aplicación resulta sencilla, pues el $84,6 \%$ de los encuestados considera que la navegabilidad es fácil o muy fácil. El $92,3 \%$ de los usuarios considera que el grado de utilidad de la aplicación es de 4 sobre 
5 o 5 sobre 5 . Los usuarios no han quedado satisfechos con la interacción por voz pues el 34,6 \% le da una puntuación media-baja. En lo que respecta a errores de la aplicación, ésta parece bastante fiable pues ninguno de los usuarios consultados ha detectados fallos al usar la aplicación, ya que el 100 \% ha marcado la casilla de "no". Concluye que la aplicación tiene una valoración bastante positiva.

\subsection{Sobre el auge de los chatbots}

Se encontraron cinco artículos que detallaban acerca del crecimiento de los agentes conversacionales. Los sistemas basados en el diálogo han existido durante un tiempo relativamente largo, y los programadores comenzaron a trabajar en el avance de los sistemas interactivos habilitados por voz desde finales de los ochenta (McTear y Griol, 2016). Sin embargo, el auge y el punto de inflexión del desarrollo de chatbot se pueden observar a partir de 2016 (McTear, 2016). Miles de desarrolladores están creando chatbots, así como herramientas de habilitación y servicios creativos en torno a los chatbots. Clasifica los casos de uso de chatbot en cuatro categorías generales: comercio colaborativo, servicio al cliente, contenido e investigación y empresa (Houston, 2017). El uso actual de chatbots es principalmente utilitario; ayudar a sus usuarios con tareas cotidianas como recordatorios de eventos del calendario, ejecutar comandos de voz mediante la redacción de un texto o un correo electrónico, responder preguntas financieras sencillas o escanear agencias de viajes para encontrar el vuelo más barato para sus próximas vacaciones (Hegna, 2018). Muchas empresas de Tl están entrando en el espacio de los chatbots, así como innumerables nuevas empresas. Algunas grandes empresas de tecnología, como Facebook y Skype, incluso decidieron reclamar a los chatbots como una herramienta comercial potencial (Onufreiv, 2017). El rápido desarrollo en esta área ha sido influenciado por varios puntos (a) avances en inteligencia artificial (IA), (b) la disponibilidad de big data, (c) mayor conectividad de dispositivos y recursos basados en la nube, (d) avances en reconocimiento de voz y procesamiento del lenguaje natural (McTear, 2017). Con la proliferación de plataformas de mensajería, los chatbots están bien posicionados para continuar tomando participación de mercado de las aplicaciones móviles a medida que brindan experiencias de usuario mejoradas (Houston, 2017). Para un mundo empresarial, donde el servicio al cliente es el factor más importante para el éxito, los chatbots podrían marcar una gran diferencia para vencer a la competencia (Onufreiv, 2017). Por último, si bien la mayoría de los chatbots pueden ser considerados utilitarios, la tecnología también tiene el potencial de ser útil en otros ámbitos sociales. Tomando a Woebot, por ejemplo, un chatbot terapéutico cognitivo-conductual que redujo significativamente los síntomas de depresión y ansiedad en sus usuarios. También hay otros bots de salud, que van desde enfermeras de chatbot que le recuerdan que debe tomar sus medicamentos, monitorear su salud y programar citas con profesionales de la salud si es necesario, hasta chatbots que 
reemplazan al asistente de un médico general, identificando condiciones probables y transmitiendo la información al médico (Hegna, 2018).

\section{DISCUSIÓN}

Este trabajo analizó la literatura científica disponible en la base de datos EBSCO y Google Académico sobre el tópico de la usabilidad percibida de los chatbots sobre la atención al cliente en las organizaciones entre 2015 y 2020. El objetivo fue realizar un análisis sobre el estado de este tópico en la base de datos mencionada. A continuación, se destacan algunos aspectos considerados importantes y se analizan algunas implicaciones para la investigación y práctica educativa.

¿Qué sabemos de la naturaleza de los chatbots? En varios estudios, los términos chatbot, agente conversacional y asistente virtual son vistos como sinónimos (Gutiérrez, 2019; Limón, 2016). Además, pocas investigaciones en nuestro estudio tratan de distinguir otro concepto, y cuando lo intentan no aportan lo suficiente como para diferenciar totalmente estos, lo que nos facilita la clarificación del término. Una característica que se refleja y se mantiene constante en todos los estudios identificados, es la actividad del chatbot como un programa informático con el que es posible mantener una conversación en lenguaje natural (Gutiérrez, 2019; Gomez, 2019; Limón, 2016; Abata y Ramírez, 2018; Herrero y Varona, 2018). De hecho, todos los artículos subrayan el papel protagonista de los chatbots en la atención al cliente. Herrero y Varona (2018) dan a entender que el estilo innovador de estos canales de comunicación, sumado al incremento de uso de redes sociales son la clave del éxito de los chatbots para comunicarse con los usuarios. Por otro lado, Gutierrez (2019) expone en su investigación acerca del reto y largo camino que aún tienen los chatbots para seguir mejorando su forma de interactuar con los usuarios.

¿Cómo puede implementarse un chatbot? La revisión de literatura nos muestra que la idea de implementar un chatbot en una organización es relativamente sencilla y dependerá de la propia organización y los objetivos que plantee. El hecho de que más del $50 \%$ de los artículos recogidos durante esta investigación se centren en el desarrollo e implementación de un chatbot nos indica la facilidad y utilidad de incorporar esta herramienta en una organización (García-Reina, 2018; Aguilar y Balbín, 2017; Cevallos y Dela, 2017; Borges, 2018). Es importante señalar que existen múltiples plataformas de desarrollo de chatbots de las cuales hacen uso muchos estudios (Expósito, 2019; Pérez, 2019; Santos, 2018; Manjarrés y Echeverri, 2020). No obstante, también es una opción válida el desarrollar un chatbot en un entorno de código libre [26,29]. No obstante, Cubero (2015) y Vallejo (2015) describen que, si bien la implementación de un chatbot es sencilla a primera vista, puede tornarse complicada al momento de integrarlo en un sistema de información que no está unificado, el cual no permitiría desarrollar todas las capacidades del chatbot correctamente. 
¿Cómo se percibe la usabilidad de los chatbots?, ¿Con qué resultados? La usabilidad percibida de los chatbots sobre la atención al cliente en las organizaciones fue tratada por un 31 \% de los artículos. En estos trabajos, los autores exponen los distintos resultados obtenidos en la implementación de un chatbot. Se evidencia que todos coinciden en concluir que efectivamente los chatbots tienen una significativa relevancia para mejorar el servicio de atención al cliente. El análisis de esta literatura muestra los múltiples indicadores usados por distintos autores para medir el rendimiento del chatbot, tales como: satisfacción, usabilidad, fiabilidad, efectividad y confiabilidad (Condori, 2017; Durán, 2015; Jimenez, 2019; Choque, 2018; Hurtado y Zúñiga, 2019). Estos indicadores son usados a elección del investigador según su estudio y preferencias. Sumado a esto, Burgos y Peralta (Burgos y Huaman, 2019; Peralta, 2018) consideran primordial medir la reducción de los tiempos de atención y la cantidad de actividades del proceso reducidas por el chatbot. Por otro lado, Estrada (2018) y Busqué (2018) exponen la importancia de la eficacia en la recepción de la información y el nivel de tolerancia a fallos que se debe tener.

¿Los chatbots se encuentran en auge? El papel de los chatbots en el mundo actual y sus futuras implicaciones son abordados por cinco artículos de esta revisión sistemática. Los artículos describen el punto de inflexión del desarrollo y expansión de los chatbots, sus aplicaciones en distintas áreas, utilidades en el servicio al cliente y potencial. Houston (2017) afirma que existe un aumento significativo en el desarrollo de los chatbots para distintas áreas. Añadiendo a esto, Onufreiv (2017) señala que muchas empresas de $\mathrm{Tl}$ están entrando en el negocio de los chatbots al reconocer su gran potencial como herramienta. Por otro lado, Hegna (2018) expone que la popularidad y rápido desarrollo de los chatbots reside en el uso del lenguaje natural para comunicarse con los usuarios e interactuar con ellos a través de una interfaz de chat o por voz. Por el contrario, Mctear (2017) considera más factores a tomar en cuenta como la mayor conectividad de dispositivos y recursos basados en la nube, los avances en inteligencia artificial y la disponibilidad de big data.

\section{CONCLUSIONES}

En esta revisión se sintetizaron los resultados de 38 estudios centrados en la usabilidad percibida de los chatbots en la atención al cliente y publicados en la base de datos Google Académico.

De la revisión, se concluye que el uso de la tecnología de chatbots en las organizaciones supone importantes mejoras para la atención al cliente. Se entiende que uno de los principales retos de los investigadores está centrado en el análisis de herramientas para el desarrollo e implementación del chatbot. Estos deben ser adaptados a las diferentes organizaciones y adaptarse a los objetivos establecidos. A medida que la literatura mundial va presentando un incremento de la comprensión 
de los chatbots, aumenta también la necesidad de presentar estudios con diseños más complejos que permitan evaluar el efecto de diferentes tipos de chatbot para promover su desarrollo. Se espera que este trabajo de sistematización contribuya a la promoción del aumento de trabajos sobre el uso de chatbots y la usabilidad brindada al servicio de atención al cliente.

\section{REFERENCIAS}

Abata Quinchuqui, C., y Ramírez Soria, P. (2018). Desarrollo de un prototipo de asistente virtual para la gestión del conocimiento de una organización [Trabajo de titulación, Universidad Politécnica Salesiana]. Repositorio Institucional Universidad Politécnica Salesiana. https://dspace.ups.edu.ec/handle/123456789/15875

Abbati Loureiro, M., y Moreno Varillas, J. L. (2019). Asistente virtual para servicios de la biblioteca de la UCM - Janet [Trabajo de fin de grado, Universidad Complutense de Madrid]. Repositorio Institucional de la UCM. https://eprints.ucm.es/id/ eprint/56669/

Aguilar Urbina, A., y Balbín Rojas A. (2017). Trabajo de arquitectura empresarial para la implementación de un chatbot en la Superintendencia de Banca, Seguros y AFP [Tesis de titulación, Universidad Peruana De Ciencias Aplicadas]. Repositorio Académico UPC. http://hdl.handle.net/10757/622652

Aquino Palacios, R., Lepage Chumpitaz, C., y Rivera Huaraca P. (2019). Solución de ChatBot aplicado a los procesos agudos en el sector Salud [Trabajo de investigación de maestría, Universidad Tecnológica del Perú]. Repositorio Institucional de la Universidad Tecnológica del Perú. https://hdl.handle.net/20.500.12867/2318

Ascencio Vargas, J. J. (2019). Diseño de un chatbot para mejorar la calidad de servicio al cliente en la empresa Fans Store S.A.C, Lima - 2019 [Trabajo de investigación de bachiller, Universidad Científica del Sur]. Repositorio Académico Universidad Científica del Sur. https://hdl.handle.net/20.500.12805/884

Borges Fernandez, E. (2018). Desarrollo de un asistente virtual conversacional proactivo basado en Dialogflow [Trabajo de fin de grado, Universidad de La Laguna]. Repositorio Institucional de la Universidad de La Laguna. http://riull.ull.es/ xmlui/handle/915/8421

Burgos Romero, M. B., y Huaman Saavedra, D. (2019). Implementación de un chatbot, utilizando la metodología ICONIX para mejorar el proceso de ventas en la empresa EAC Steel E.I.R.L [Tesis de titulación, Universidad Autónoma del Perú]. Repositorio de la Universidad Autónoma del Perú. http://repositorio.autonoma.edu.pe/ handle/AUTONOMA/852 
Busqué Somacarrera, V. (2018). Asistente virtual para estudiantes de la FIB [Trabajo de fin de grado]. Universitat Politècnica de Catalunya. http://hdl.handle.et/2117/ 122656

Durán Pincheira, A. D. (2015). Diseño E Implantación De Un Asesor Virtual Con Interfaz Web Basado En Un Sistema De Gestión De Conocimientos Y Autoaprendizaje [Tesis de titulación, Universidad de las Fuerzas Armadas]. Repositorio Institucional de la Universidad de las Fuerzas Armadas ESPE. http://repositorio.espe.edu.ec/ handle/21000/10297

Cevallos Tóala, M. A., y Dela Indio, J. T. (2017). Propuesta tecnológica de una página web con la implementación de bots para la gestión de relaciones con el cliente en la empresa Vipcell Electronics [Tesis de titulación, Universidad de Guayaquil]. Repositorio Universidad de Guayaquil. http://repositorio.ug.edu.ec/handle/redug/21898

Choque Díaz, M. L. (2018). Chatbot académico utilizando tecnologías cognitivas. [Tesis de titulación, Universidad Peruana De Ciencias Aplicadas]. Repositorio Académico UPC. https://doi.org/10.19083/tesis/624418

Condori Quispe, W. (2017). Desarrollo de un Asistente Virtual Utilizando Facebook Messenger para la Mejora del Servicio de Atención al Cliente en la Universidad Privada de Tacna en el 2017 [Tesis de titulación, Universidad Privada de Tacna]. Repositorio de la Universidad Privada de Tacna. http://repositorio.upt.edu.pe/ handle/20.500.12969/163

Cubero Caba, L. E. (2015). Asistente Virtual (Chatbot) para la web de la Facultad de Informática [Trabajo de curso]. Universidad Complutense de Madrid. https://eprints.ucm.es/ id/eprint/32448/

Díaz Garcia, R. (2020). Asistente virtual conversacional aplicado al ámbito cultural. http:// hdl.handle.net/10016/31688

Estrada Cutimbo, L. (2018). Implementar chatbot basado en inteligencia artificial para la gestión de requerimientos e incidentes en una empresa de seguros [Tesis de titulación, Universidad San Ignacio de Loyola]. Repositorio Institucional de la Universidad San Ignacio de Loyola. http://repositorio.usil.edu.pe/handle/ USIL/8844

Expósito García, Y. (2019). Bullbot: Asistente Virtual para la Gestión de Servicios de la Biblioteca de la ULL [Trabajo de fin de grado, Universidad de La Laguna]. Repositorio Institucional de la Universidad de La Laguna. http://riull.ull.es/ xmlui/handle/915/14747

García-Reina, L. F. (2018). Asistente virtual de tipo ChatBot. Universidad Católica de Colombia. https://repository.ucatolica.edu.co/handle/10983/17726 
Garibay Ornelas, F. A. (2020). Diseño e implementación de un asistente virtual (chatbot) para ofrecer atención a los clientes de una aerolínea mexicana por medio de sus canales conversacionales [Proyecto de maestría]. INFOTEC Centro de Investigación e Innovación en Tecnologías de la Información y Comunicación. https://infotec. repositorioinstitucional.mx/jspui/bitstream/1027/402/1/INFOTEC_MGITIC_ FAG0_27082020.pdf

Gómez Loizaga, H. (2019). Agente conversacional para búsqueda y recomendación de ofertas laborales [Tesis de maestría, Universidad Internacional de la Rioja]. Repositorio Institucional de la Universidad Internacional de La Rioja. https://reunir.unir.net/ handle/123456789/9564

Guijarro Marco, P. (2020). Asistente virtual para un sistema de información [Trabajo de fin de grado, Universidad de Alicante]. Repositorio Institucional de la Universidad de Alicante. http://hdl.handle.net/10045/101934

Gutiérrez Siliceo, J. M. (2019). Desarrollo de chatbots con entornos de código abierto [Tesis de titulación, Universidad De Cantabria]. Repositorio Abierto de la Universidad De Cantabria. http://hdl.handle.net/10902/16456

Hegna Berge, S. (2018). Rise of the Chatbots: Trust in Artificial Intelligence During Extreme Weather Events [Master's Thesis, University Of Oslo]. University of Oslo Library. http://urn.nb.no/URN:NBN:no-65934

Herrero Diz, P., y Varona Aramburu, D. (2018). Uso de chatbots para automatizar la información en los medios españoles. El profesional de la información, 27(4), 742-749. https://doi.org/10.3145/epi.2018.jul.03

Houston J. (2017). The Rise of Chatbots!: Identifying Winners in the Next Wave of HumanTechnology Interaction. Navidar. https://www.navidar.com/wp-content/uploads/ 2017/01/Chatbots_Article-5.24.17.pdf

Hurtado Moína, K. L., y Zúñiga Loaiza, J. I. (2019). Desarrollo de un asistente virtual web para la EPN y un asistente dirigido por voz en los kioscos digitales de la DGIP [Trabajo de titulación, Escuela Politécnica Nacional] Repositorio Digital Institucional de la Escuela Politécnica Nacional. http://bibdigital.epn.edu.ec/ handle/15000/20051

Jimenez Flores, V. J. (2019). Entidad conversacional de inteligencia artificial y calidad del servicio percibido por estudiantes de la Universidad José Carlos Mariátegui Filial Tacna, 2018-II [Tesis de titulación, Universidad Nacional Jorge Basadre Grohmann]. Repositorio Institucional Digital de la Universidad Nacional Jorge Basadre Grohmann. http://repositorio.unjbg.edu.pe/handle/UNJBG/3848 
Limón Pérez, M. A. (2016). Construcción de un prototipo de programa personalizado de tipo chatbot en ambiente java con un lenguaje natural [Tesis de titulación]. Instituto Politécnico Nacional. http://tesis.ipn.mx:8080/xmlui/handle/123456789/17959

Manjarrés Betancur, R. A., y Echeverri Torres, M. M. (2020). Asistente virtual académico utilizando tecnologías cognitivas de procesamiento de lenguaje natural. Revista Politécnica, 16(31), 85-96. https://doi.org/10.33571/rpolitec.v16n31a7

Martínez Carpio, J. A. (2019). Desarrollo de un asistente virtual (chatbot) para la automatización de la atención al cliente [Tesis de titulación, Universidad de Guayaquil]. Repositorio Universidad de Guayaquil. http://repositorio.ug.edu.ec/ handle/redug/45108

McTear, M., Callejas, Z. y Griol, D. (2016). The Conversational Interface: Talking to Smart Devices. Springer International Publishing. https://doi.org/10.1007/978-3-31932967-3

McTear, M. F. (2017). The rise of the conversational interface: A new kid on the block? In J. Quesada, F. Martín, T. López (Eds.), Future and Emerging Trends in Language Technology. Machine Learning and Big Data (pp. 38-49). Springer. https://doi. org/10.1007/978-3-319-69365-1_3

Onufreiv, Y. Y. (2017). The Rise in Chatbots Trends. National Aviation University of Ukraine. http://er.nau.edu.ua/handle/NAU/27919

Peralta Pinedo, A. G. (2018). Chatbot para la asistencia personalizada en el proceso de obtención de título en la modalidad de tesis para los bachilleres de la escuela profesional de ingeniería de computación y sistemas de la UPAO. [Tesis de Titulación, Universidad Privada Antenor Orrego]. Repositorio Digital de la Universidad Privada Antenor Orrego. https://hdl.handle.net/20.500.12759/4664

Pérez Vallejo, P. J. (2019). Implementación de un agente conversacional para negocio de repuestos automotrices integrado a plataformas de mensajería instantánea. [Tesis de titulación]. Pontificia Universidad Católica Del Ecuador.

Pérez Vila, M. A. (2019). Asistente Virtual para sitios web [Trabajo de fin de grado, Universidade Da Coruña]. Repositorio Institucional de la UDC. http://hdl.handle. net/2183/24908

Santos Buitrago, R. (2018). Desarrollo de un Asistente Virtual turístico para la ciudad de Madrid [Trabajo de fin de grado, Universidad Complutense de Madrid]. Repositorio Institucional de la UCM. https://eprints.ucm.es/id/eprint/48836/

Urrutia, G., y Bonfill, X. (2010). Declaración PRISMA: Una propuesta para mejorar la publicación de revisiones sistemáticas y metaanálisis. Medicina Clínica, 135(11), 507-511. 
https://bmjopen.bmj.com/content/bmjopen/suppl/2013/06/10/bmjopen2012-002330.DC1/bmjopen-2012-002330supp_PRISMA-2010.pdf

Vallejo Ruiz, I. (2015). Asistente virtual (chatbot) para la web de la Facultad de Informática [Trabajo de fin de grado, Universidad Complutense de Madrid]. Repositorio Institucional de la UCM. https://eprints.ucm.es/id/eprint/33443/

Zarabia Zúñiga, O. H. (2018). Implementación de un chatbot con botframework: caso de estudio, servicios a clientes del área de fianzas de seguros Equinoccial [Tesis de titulación, Escuela Politécnica Nacional]. Repositorio Digital Institucional de la Escuela Politécnica Nacional. http://bibdigital.epn.edu.ec/handle/15000/19628 